\title{
Thyroid Hormone Tests Ordering Practice and Cost-Effectiveness in Samples Referred to International Clinical Laboratories from Addis Ababa Health Facilities
}

\author{
Kifle Tilahun ${ }^{1}$, Meaza Demissie ${ }^{2}$, Tamrat Bekele ${ }^{3}$, Mesfin Nigussie ${ }^{4}$, Damen \\ H/Mariam ${ }^{5}$
}

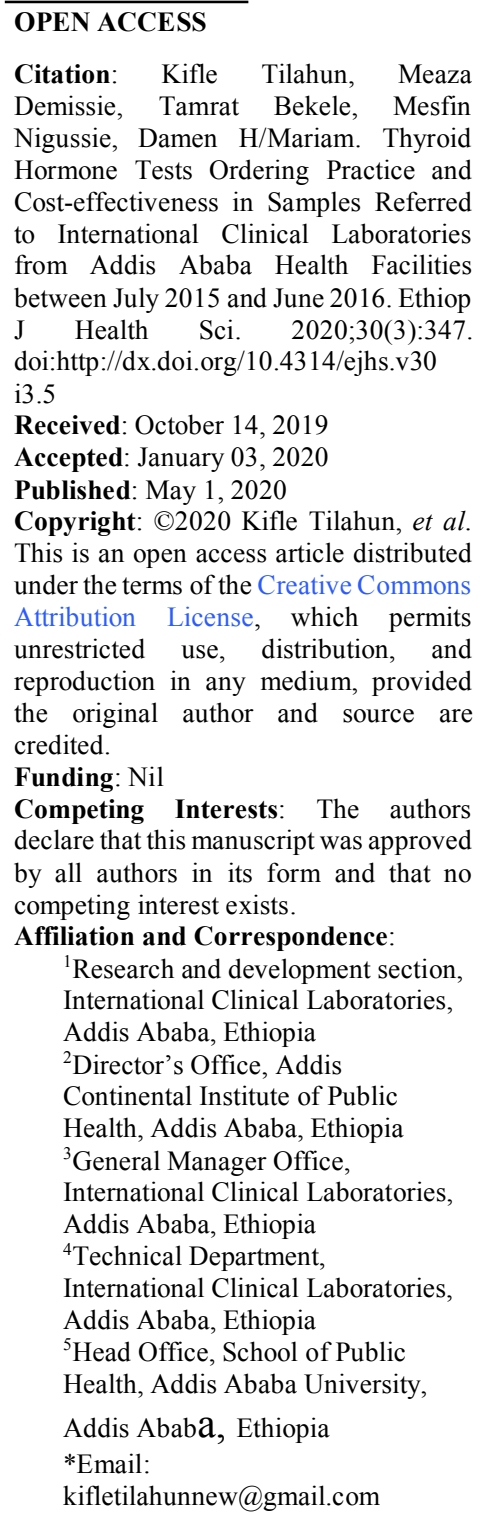

\begin{abstract}
BACKGROUND: Thyroid dysfunction accounts for majority of endocrine disorders. In sub-Saharan Africa Graves' disease and hypothyroidism have accounted for $13.1 \%$ and $8.8 \%$ while the burden of thyroid disorder has ranged from 6.18 to47.34\% among countries in the Arab world. The cost for a primary thyroid test done to evaluate the gland function constituted a large proportion of the public health budget. For instance, 10 million thyroid functions have been done each year by laboratories which cost 30 million $U K$ pounds, and they represent $8 \%$ of laboratory charge in the US. When a TSH-only protocol (guideline) was used, 95\% of the requests were sufficient for diagnosis without requiring further tests, thereby resulting in 50\% savings on FT4 reagent and reducing the annual TFT reagent cost by $25 \%$. This is an original study, and its objective was to assess the ordering pattern of TSH tests and their cost-effectiveness in patients' samples referred to ICL from Addis Ababa health facilities between July2015 to June 2016
\end{abstract}

METHOD: An institution-based cross-sectional study design was utilized to study the ordering pattern of thyroid function tests using one-year retrospective data from ICL.

RESULTS: Thyroid profiles were ordered more frequently (49.5\%) compared to TSH only (24.3\%). An additional 2625.70 USD was paid by patients for individual components in the profile tests that turned out normal.

CONCLUSION: Guidelines advocate TSH as the initial test for thyroid dysfunction, but the use of a combination of tests is more common.

KEYWORDS: Thyroid stimulating hormone, Thyroid dysfunction. Cascade testing.

\section{INTRODUCTION}

Thyroid dysfunction accounts for the majority of endocrine disorders (1). In sub-Saharan Africa, Graves' disease and hypothyroidism have accounted for $13.1 \%$ and $8.8 \%$ while the burden of thyroid disorder has ranged from 6.18 to $47.34 \%$ among countries in the Arab world 
$(2,3)$. Endemic goiter has been found in $28.6 \%$ in the community of Sekota, Ethiopia (3). The cost for a primary thyroid test done to evaluate the gland function constituted a large proportion of the public health budget (4). For instance, 10 million thyroid functions have been done each year by laboratories which cost 30 million UK pounds, and they represent $8 \%$ of laboratory charge in the US (5).

$\mathrm{TSH}$ measurement is a reliable test to diagnose all common forms of hypothyroidism and hyperthyroidism. Pituitary gland controls T4 (thyroxin) and T3 (triiodothyronine) secretion with a corresponding negative feedback release of TSH by the pituitary $(6,7)$ Because of this log-linear relationship between the serum levels of T4, T3 and the production of TSH by the pituitary gland, minute changes in serum thyroxin and triiodothyronine have been found to induce significant changes in the serum level of TSH. This implies that TSH can identify slight changes in thyroid hormone production and is therefore the best indicator of thyroid function (8). In normal individuals, when the TSH was within the reference range, there was a $99 \%$ likelihood that the FT4 will also be within the reference range (9) (1). Various standard guidelines to evaluate thyroid function have been developed based on the above phenomena $(9,10)$. These algorithms and guidelines apply a cascade testing procedure to effectively evaluate functional thyroid status while avoiding extra laboratory costs (11). The cascade begins with a TSH test. If a patient has a normal pituitary-thyroid axis, the TSH level indicates normal physiologic thyroid hormone activity (12).

Guidelines that advocate cascade testing have multiple advantages than ordering the combination of tests. However they are not appropriately and uniformly practiced by physicians (13).

Costs from such unnecessary routine requests of thyroid function tests (TFTs) are increasing, as indicated by studies that showed that the frequency of ordering TFT is higher than that for the TSH only test. The cost of performing a TFTs test is substantially higher than that for the TSH only test $(14,15)$.

Consequently, the cost for a primary thyroid test to evaluate gland function constituted a large proportion of the public health budget (4). For instance, 10 million thyroid function tests are performed each year by laboratories at a cost of 30 million UK pounds; these tests also represent 8\% of laboratory charges in the US (5). A study at a student clinic showed that the cost of TFTs could be reduced ten-fold if the standard diagnostic protocol were used (16). Similarly, when a TSHonly protocol (guideline) was used, 95\% of the requests were sufficient for diagnosis without requiring further tests, thereby resulting in a $50 \%$ savings on FT4 reagent and reducing the annual TFT reagent cost by $25 \%$ (6). No similar study has been done in Ethiopia. This is an original study. The objectives were i) to assess ordering pattern of TSH tests performed between July 2015 and June 2016, and ii) to determine the unnecessary cost of thyroid function tests performed between July 2015 and June 2016. Results were compared with the current guidelines, and cost effectiveness was analyzed.

\section{MATERIALS AND METHODS}

The study was conducted in International Clinical Laboratory (ICL) Addis Ababa, Ethiopia. An institution-based cross-sectional study design was utilized to study the ordering pattern of thyroid function tests using one-year retrospective data from the ICL.

The study sources were records of all blood samples that were analyzed at the immunoassay system of the International Clinical Laboratories between July 2015 and June 2016 for serum triiodothyronine (T3), thyroxine (T4), thyroidstimulating hormone (TSH), combined thyroid function tests including (T3+T4), (TSH+T4), $(\mathrm{TSH}+\mathrm{T} 3+\mathrm{T} 4)$ and others.

TSH, T3, T4, FT4 and FT3 were the assays performed. Determination of serum values was performed on an automated immunoassay analyzer (AIA), which functions according to the principle of fluoroimmunoassay. The normal reference ranges for variables were as follows: $\mathrm{T} 3=0.58$ $1.59 \mathrm{ng} / \mathrm{ml}, \quad \mathrm{T} 4=4.8-11.72 \mu \mathrm{g} / \mathrm{dl}, \quad$ FT3 $=1.71-$ $3.71 \mathrm{pg} / \mathrm{ml}, \quad \mathrm{FT} 4=0.70-1.48 \mathrm{ng} / \mathrm{dl}$ and $\mathrm{TSH}=0.35-4.94 \mu \mathrm{IU} / \mathrm{ml}$.

In the study period, from July 2015 to May 2016, the immunoassay unit of the ICL performed 15,635 thyroid function tests from various parts of the country. Among these, 8,313 were from health facilities in Addis Ababa.

An institution-based study conducted in India by Shalini Gupta to represented $46 \%$ of all thyroid tests performed (7). No similar studies for this proportion have been done in Ethiopia. This 46\% 
proportion was used to calculate the sample size using the formula for a single population proportion with an assumed $95 \%$ confidence interval and 5\% margin of error and, proportion of TSH tests done out of all forms of thyroid tests $(p=0.46$.). The outcome was 382 .The 382 tests from the Addis Ababa requests were selected from the 8313 tests in ICL as follows:

Sampling procedure: Systematic random sampling method was applied to select the sample. Records of all immunoassay tests performed between July 2015 and June 2016 was used as data source. Records of all single as well as combined thyroid function tests performed during the same period was used as sampling frame. The first date of sample collection was randomly chosen from a lottery that contains the dates of July 1 to July 10 of the year 2015.

Then, every tenth sample was taken until thirty-two or thirty-one thyroid tests were obtained in thirty days. This procedure was repeated on total thyroid tests performed monthly until the desired sample, 382, was achieved in the total 12 months between July 2015 and June 2016. The tenth sample was chosen based on the observation that a minimum of 300 tests are performed every month which will allow the collection of the desired sample within the intended study period while allowing the sample collection to spread through twelve months. This allowed the study to cover and hence reflect on a one year test ordering practice.

Data were entered and processed with SPSS statistical software to analyze the descriptive statistics i) for different combinations of thyroid tests ordered, ii) to determine the percentage of TSH and TFT tests ordered, and iii) to obtain the results of thyroid profile samples as well as thyroid hormone tests.

An analysis of indirect and opportunity costs was not performed. Analysis of the direct costs incurred by ordering all forms of TFT and standard TSH test alone was made by simple accounting. Similarly, the direct cost of health care expenditure for diagnostic testing that can be saved was performed by simple cost analysis.

Ethical approval was obtained from the Internal Review Board of the Addis Continental Institute of Public Health. After review, the ethical clearance letter was submitted to Addis Ababa Health Bureau, where it was reviewed and approved. Data collection was performed after obtaining permission from ICL.

\section{RESULTS}

Among 8313 thyroid function tests referred to ICL from health facilities in Addis Ababa, 382 requests were selected for this study. Maximum samples were requested for age group 31 to 40 for both sexes (Table 1). Combined thyroid function tests containing TSH were included in $75.7 \%$ of the samples and thyroid stimulating hormone only test was performed in $24.3 \%$ (Figure 1).

analyze the ordering pattern of thyroid-related tests, referred by clinicians to an endocrine laboratory, showed that TSH tests

Table 1: Sex by age distribution of patients for whom thyroid function tests were performed at ICL between July 2015 and June 2016: Page 11, line\# 3 \&5, Results section

\begin{tabular}{|c|c|c|c|c|c|c|c|c|c|c|c|c|c|}
\hline $\begin{array}{l}\text { Age } \\
\text { years }\end{array}$ & in & $<1$ & $\begin{array}{l}\text { 1to1 } \\
0\end{array}$ & $\begin{array}{l}\text { 11to2 } \\
0\end{array}$ & $\begin{array}{l}\text { 21to3 } \\
0\end{array}$ & 31to40 & $\begin{array}{l}\text { 41to5 } \\
0\end{array}$ & $\begin{array}{l}\text { 51to6 } \\
0\end{array}$ & $\begin{array}{l}\text { 61to7 } \\
0\end{array}$ & $\begin{array}{l}71 \text { to8 } \\
0\end{array}$ & $\begin{array}{l}81 \text { to } 9 \\
0\end{array}$ & $91+$ & Total \\
\hline \multicolumn{14}{|l|}{ Sex } \\
\hline Male & & 3 & 6 & 3 & 15 & 20 & 21 & 17 & 10 & 6 & 3 & 1 & 105 \\
\hline \multirow{3}{*}{ Female } & & $0.8 \%$ & $1.6 \%$ & $0.8 \%$ & 3.9 & $5.2 \%$ & $5.50 \%$ & $4.5 \%$ & $2.60 \%$ & $1.6 \%$ & $0.8 \%$ & $0.3 \%$ & $27 \%$ \\
\hline & & 3 & 7 & 17 & 60 & 68 & 52 & 39 & 24 & 7 & 0 & 0 & 277 \\
\hline & & $0.8 \%$ & $1.8 \%$ & $4.5 \%$ & $15.7 \%$ & $\begin{array}{l}17.80 \\
\%\end{array}$ & $13.6 \%$ & $10.2 \%$ & $10.3 \%$ & $1.8 \%$ & $0.0 \%$ & 0 & $\begin{array}{l}72.5 \\
\%\end{array}$ \\
\hline \multirow{2}{*}{\multicolumn{2}{|c|}{ Total }} & 6 & 13 & 20 & 75 & 88 & 73 & 56 & 34 & 13 & 3 & 1 & 382 \\
\hline & & $1.6 \%$ & $\begin{array}{l}3.4 \\
\%\end{array}$ & $5.2 \%$ & $\begin{array}{l}19.6 \\
\%\end{array}$ & $23.0 \%$ & $\begin{array}{l}19.1 \\
\%\end{array}$ & $\begin{array}{l}14.7 \\
\%\end{array}$ & $\begin{array}{l}8.90 \\
\%\end{array}$ & $3.4 \%$ & $0.8 \%$ & $\begin{array}{l}0.3 \\
\% \\
\end{array}$ & $\begin{array}{l}100 \\
\%\end{array}$ \\
\hline
\end{tabular}




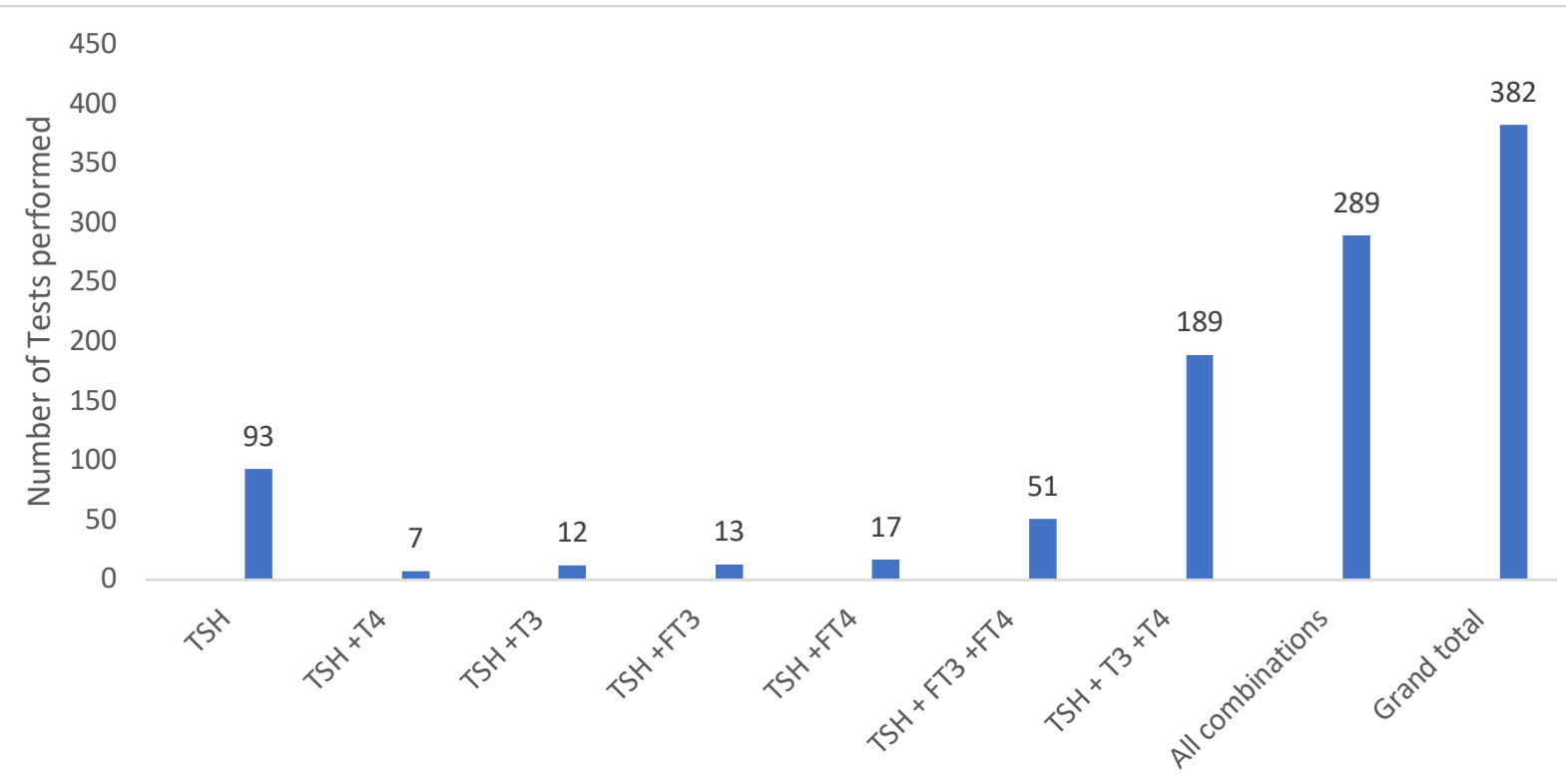

Number of Tests performed

Figure 1: Analysis of ordering pattern for different combinations of thyroid tests performed at ICL between July 2015 and June 2016; Page 11 Line \#5, Results section

All of the 382 requests contained TSH tests. Out of the total $382 \mathrm{TSH}$ tests performed in the form of TSH only requests, TSH included in panel (TSH+T3+T4) and in other combinations; $72.8 \%$ samples had results within the reference interval.
Twelve percent of samples had TSH results higher and $15.2 \%$ had values lower than the reference range. The proportion of TSH samples with normal results was $78.49 \%$ among $\mathrm{TSH}$ only requests while $70.93 \%$ of TSH in the combination samples had their values in the reference range (Table 2).

Table 2: Analysis of results of TSH samples performed at ICL between July 2015 and June 2016 Page 11 line\# 111 and 13 ,Results section

\begin{tabular}{|c|c|c|c|c|c|}
\hline $\begin{array}{l}\text { Type sample TSH test is } \\
\text { requested in }\end{array}$ & $\begin{array}{l}\text { Resul } \\
\text { t of } \\
\text { TSH } \\
\text { test } \\
\end{array}$ & $\begin{array}{l}\text { Number of } \\
\text { tests } \\
\text { with normal } \\
\text { TSH }\end{array}$ & $\begin{array}{l}\text { Number of } \\
\text { tests } \\
\text { with High } \\
\text { TSH }\end{array}$ & $\begin{array}{l}\text { Number of } \\
\text { tests } \\
\text { with Low } \\
\text { TSH }\end{array}$ & Total \\
\hline TSH only samples & & $73(78.49 \%)$ & $8(8.6 \%)$ & $12(12.9 \%)$ & $93(100 \%)$ \\
\hline $\begin{array}{l}\text { Total TSH (all TSH tests } \\
\text { found } \\
\text { in all types of combined } \\
\text { samples) }\end{array}$ & & $205(70.93 \%)$ & $38(13.14 \%)$ & $46(15.91 \%)$ & $289(100 \%)$ \\
\hline Total & & $278(72.8 \%)$ & $46(12.0 \%)$ & $58(15.2 \%)$ & $382(100.0 \%)$ \\
\hline
\end{tabular}

The total number of combination tests performed were 289 . Of these, $64.7 \%$ had results within the normal reference range while $35.3 \%$ had results above and below the normal range (Table 2). The combination test containing FT4 and TSH had the highest proportion, $88.23 \%$ while $59 \%$ of the 
combination test containing TSH, FT3 and FT4 had values within the reference range (Table 3 ).

There were 529 individual tests (T3, T4, FT3, and FT4) components of the profile and combination tests. The majority of these tests $(86.2 \%)$ turned out to be normal, while $9.1 \%$ were above and $4.7 \%$ were below the reference range (Table 4).

Table 3. Analysis of results of profile (combination) tests performed at ICL between July 2015 and June 2016; Page 8, Line \#17, Results section.

\begin{tabular}{llllll}
\hline Test & \multicolumn{2}{l}{ Normal results } & \multicolumn{2}{l}{ Abnormal results } & Total \\
\hline TSH+T3+T4 & 120 & $(63.49 \%)$ & 69 & $(36.51 \%)$ & 189 \\
TSH+FT3+FT4 & 30 & $(59 \%)$ & 21 & $(41 \%)$ & 51 \\
TSH+T3 & 9 & $(75.00 \%)$ & 3 & $(25 \%)$ & 12 \\
TSH+T4 & 6 & $(85.70 \%)$ & 1 & $(14.30 \%)$ & 7 \\
TSH+FT3 & 7 & $(53.84 \%)$ & 6 & $(46.16 \%)$ & 13 \\
TSH+FT4 & 15 & $(88.23 \%)$ & 2 & $(11.77 \%)$ & 17 \\
\hline Total & 187 & $64.70 \%$ & 102 & $(35.30 \%)$ & 289 \\
\hline
\end{tabular}

Table 4: Analysis of results of individual tests included in profile / combination samples performed at ICL between July 2015 and June 2016; Page 12, line \# 3

\begin{tabular}{lllll}
\hline Test type & Normal & High & Low & Total \\
\hline FT3 & $52(81.2)$ & $8(12.5 \%)$ & $4(6.2 \%)$ & $64(100.0 \%)$ \\
FT4 & $58(81.2)$ & $7(12.5)$ & $3(6.2)$ & $68(100.0 \%)$ \\
T3 & $175(87.1 \%)$ & $23(11.4 \%)$ & $3(1.5 \%)$ & $201(100.0 \%)$ \\
T4 & $171(87.2 \%)$ & $10(5.1 \%)$ & $15(7.7 \%)$ & $196(100.0 \%)$ \\
\hline Total & $456(86.2 \%)$ & $48(9.1 \%)$ & $25(4.7 \%)$ & $529(100.0 \%)$ \\
\hline
\end{tabular}

The difference in Ethiopian Birr between the price for each combination test and that of TSH only test ranged from 170 to $375 \mathrm{Eth} \mathrm{Br}$ (7.4-16.2USD). This was used to calculate extra expenditure by patients for having the combination tests that had their results within reference range for all parameters performed (Table 5).

The amount of money spent on combined or panel tests that turned out normal was 60260 Ethiopian Birr (2625.70USD). The TSH tests done along with each of these tests were also in the normal range. The shown amount does not include the money patients pay for the TSH test.This money is paid by patients to have the individual components in the profile tests performed (Table $5)$.
Thyroid profile had the highest proportion in which 41400 Eth. Birr (1803 USD) was spent on tests with normal values the diagnosis of which could have been made by performing TSH only tests. The combination test TSH+FT3+FT4 was second causing an unnecessary expenditure of 1125 Eth. Birr (490 USD). The total amount of expenditure incurred on the 187 patients was 60260 Eth. Birr (2625 US) (Table 5).

The amount of expenditure on reagents for performing the same tests was calculated in the same manner as for the direct cost for patients. As presented in table 5 it was 18,853 Eth. Birr (821.48USD). This was an additional reagent cost to the company due to each combination test ranged was 49 to 116 Eth. Birr according to the type of test ordered. 
Table 5: Unnecessary expenditure by patients for individual tests included in a combination request that had normal results at ICL during July 2015 and June 2016; Page 12, line \#7, 11, 16 Results section and Page 16, line 12, Discussion section.

\begin{tabular}{llllllll}
\hline & $\begin{array}{l}\text { Number } \\
\text { of } \\
\text { normal } \\
\text { tests }\end{array}$ & $\begin{array}{l}\text { Single test } \\
\text { price }\end{array}$ & $\begin{array}{l}\text { Total } \\
\text { combined } \\
\text { test price }\end{array}$ & $\begin{array}{l}\text { TSH } \\
\text { single } \\
\text { Price }\end{array}$ & $\begin{array}{l}\text { Total TSH } \\
\text { test } \\
\text { Price }\end{array}$ & $\begin{array}{l}\text { Difference in } \\
\text { price } \\
\text { for single test }\end{array}$ & $\begin{array}{l}\text { Sum of } \\
\text { the } \\
\text { differen } \\
\text { ce }\end{array}$ \\
\hline TSH+T3+T4 & 120 & 520 & 62400 & 175 & 21000 & 345 & 41400 \\
TSH+FT3+F & & & & & & & \\
T4 & 30 & 550 & 16500 & 175 & 5250 & 375 & 11250 \\
TSH+T3 & 9 & 345 & 3105 & 175 & 1575 & 170 & 1530 \\
TSH+T4 & 6 & 345 & 2070 & 175 & 1050 & 170 & 1020 \\
TSH+FT3 & 7 & 405 & 2835 & 175 & 1225 & 230 & 1610 \\
TSH+FT4 & 15 & 405 & 6075 & 175 & 2625 & 230 & 3450 \\
\hline Total & $\mathbf{1 8 7}$ & & $\mathbf{9 2 9 8 5}$ & & $\mathbf{3 2 7 2 5}$ & & $\mathbf{6 0 2 6 0}$ \\
\hline
\end{tabular}

\section{DISCUSSION}

In this study, the TSH only test accounted for only one-fourth and combination (profile) samples for three-fourths of the requests, while the opposite result was expected according to the standard guidelines to evaluate thyroid function. An unnecessary 60260 Ethiopian Birr (2625.70 USD) and 18853 Ethiopian Birr (821.48 USD) additional cost was incurred on patients and health facilities respectively, due to ordering a high proportion of profile or combination tests by clinicians. This shows that although the guidelines favor TSH as the screening test for thyroid dysfunction, the use of combination tests is more common.

Apart from a few cases, the results of the combination TFT were not remarkable or did not have abnormal results. The findings of the study which revealed that $78 \%$ of TSH tests, 65 to $88 \%$ of combined tests, as well as $86 \%$ of thyroid hormone tests to have normal results also suggests that the majority of the cases could have been diagnosed by clinical impression only. The observation that almost half of the requests were for profile tests was similar to a study in India by Shalini Gupta et al where $47.5 \%$ of requests were for profile samples (6). It can be observed that the majority of the clinicians are not sticking to cascade testing for thyroid function tests.

There was no significant difference in the proportion of normal TSH levels when all the TSH tests done during the study period were taken into consideration: TSH only tests had normal results in $78.49 \%$ of tests, while $70.9 \%$ of TSH included in combined tests had results in the reference range. This is similar to the finding of $79.2 \%$ in the study by Shalini Gupta et al (6). This can imply that estimation of thyroid hormones gives no additional information in the majority of patients other than confirmation of normality.

The majority (65\%) of combination tests had results within the reference interval for all the three parameters and thus excluded thyroid disorder making the likelihood of thyroid dysfunction around one in every seven patients presumed to have it. This finding is in conformity with the existing knowledge that $99 \%$ of blood tests for thyroid hormones fall within normal range if TSH is normal $(9,17)$. This implies the thyroid function status of these patients with normal results for all parameters could have easily been determined by performing TSH only tests alone. This finding is similar but a little lower than the proportion in a study by Shalini et al in which the percentage was around $77 \%$ (6).

The fact that the majority of the individual tests (T3, T4, FT3 and FT4) turned out to be normal $(86.2 \%)$ which rules out presence of thyroid dysfunction indicates indiscriminate test ordering. This is in line with similar study by Shalini Gupta et al in which $86.2 \%$ were normal (6).

An analysis of the patient and reagent cost was used to estimate the difference in expenditure between ordering only the TSH test and combination tests. The majority of the combination requests had results within the normal range. This implies that adding the same number of tests to the TSH in each combination could have been avoided if the cascade testing was applied in which TSH is ordered. Likewise, significant material and time 
resources could have been saved. This is further highlighted by the fact that these tests are not available everywhere and that the few facilities performing these tests are burdened with an unnecessary workload.

Thyroid profile $(\mathrm{TSH}+\mathrm{T} 3+\mathrm{T} 4)$ had the highest proportion, and 41,400 Ethiopian Birr (1,803 USD) was spent on tests with normal values for which the diagnosis could have been made by performing only a TSH test. The combination test TSH+FT3+FT4 was second, causing an unnecessary expenditure of 11,250 Ethiopian Birr (490 USD). The total amount of expenditure incurred on the 187 patients was 60,260 Ethiopian Birr (2,625 USD) (Table 5). These funds could have been saved if only a TSH test was ordered for these patients. A similar observation was made by Kawfehr El Shafe et al. in a study in Oman in which only $6 \%$ of the tests administered had positive results, resulting in an unnecessary TFT expenditure amounting to 18,800 USD. A similar result was also found in a study by Anthony Viera et al $(16,18)$.

The expenditure on reagent for the same number of tests was 18853 Eth. Birr (821 USD). This money could have been spent for procurement of other reagents for tests that are of high demand. The cost-savings reported here are total incremental direct costs (reagents and labor). This finding is in line in its context with a study by Anthony Viera et al in which it was shown that if combination test had not been ordered on the 1,192 specimens with normal TSH results, the actual material cost savings over the 26-month study period would have been more than $\$ 3,360$ (18).

A five-year data of blood tests for thyroid function at ICL was analyzed for further inference. In the five years, 47144 all forms of thyroid function tests were performed of which TSH only tests accounted for $25 \%$ while $75 \%$ were combined tests. A large majority (75\%) of the requests were for combination tests. This is in conformity with the finding of this study on the 382 samples in which $75.7 \%$ of the blood tests ordered were for combination tests. This again demonstrates that clinicians in health facilities that refer samples to ICL prefer to order combination/profile thyroid tests than TSH tests as opposed to the recommended teaching of using algorithm or cascade testing. This finding is even greater than the observation of a study in India by Shalini Gupta in which TFT was ordered in 54\% of cases (6).

Analysis of cost was made on the combination tests performed in the five years. The percentage of normal tests among each combination test was used to make extrapolation of expenditure by patients for whom the combination tests were performed.

Projected cost savings calculation was based on initial observations in the current study. Overall, $63.5 \%$ of profile (TSH+T3+T4), $59 \%$ of (TSH+FT3+FT4), and $75 \%$ of $(\mathrm{TSH}+\mathrm{T} 3)$ were normal. .Likewise $85.7 \%$ of (TSH+T4), $53.8 \%$ of (TSH+FT3) and $88.2 \%$ of (TSH+FT4) requests had results within the normal range. By this projection 22560 of the 35360 requests were for $\mathrm{TSH}+\mathrm{T} 3+\mathrm{T} 4$ test.

In the current study, the percentage of tests with normal results for this combination was $63.49 \%$ which makes the number of normal tests 14325.6 out of the 22560. The price for $\mathrm{TSH}+\mathrm{T} 3+\mathrm{T} 4$ test is 520 Eth. Birr while that of single TSH test is 175 and this makes the extra payment 345 Eth. Birr for a single test. Multiplying this difference,(345EthBirr), by the number of normal tests in 5 years which were 14325.6 amounts to 4,942,332 Eth. Birr (215,352.15 USD). This is the extra financial burden incurred on patients due to all $\mathrm{TSH}+\mathrm{T} 3+\mathrm{T} 4$ tests with normal results in the five year period. Likewise, the total expenditure that could have been saved by initially requesting TSH only tests in each of the six types of the combination requests was analyzed and the sum total amounted to 7,417,413.10 Eth. Birr (323,198.82 USD).

The cost of the reagent required to perform these tests was 2,339,745.70Eth. Birr $(101,949.70$ USD). This implies a total of 9,757,158.80 Eth. Birr (425,148,53 USD) could have been saved and used for other purposes if an initial TSH only test was ordered for these patients as per the guideline. This finding is consistent with the study by Geoff Beckett et al who found that 30 million pounds were spent on TFT each year in the UK (5).

The most significant conclusion that can be made is that the use of thyroid profile and other combination tests is common and results in an extra financial burden for patients and the healthcare system. This study had large sample size which was adequate for statistical inference and projection. Data were complete for all variables and were from an 
internationally accredited laboratory. Lack of data on specific clinical reason for requesting combination tests as the requests had no indication for the tests.

\section{REFERENCES}

1. B, Bartalena L, Cooper D.S., Hegedus L, Laurberg P, Kahaly G.j. Eur Thyroid J 2015;4:149-163.

2. Awad Saad Al Shahrani, El-Metwally Ashraf, Khaled Al - Surimi (last), Salih SB, Saleh Yousef, Abeer $\mathrm{Al}$, et al. The epidemiology of thyroid disease in the Arab world: A systematic review. $J$ Public health \& epidemiol.ISSN; 2141-2316 2016 Feb; 8(2):17-26.

3. El Hassane Sidibe. Thyroid diseases in SubSaharan Africa. John Libbey Eurotext 2006 Nov;17(1):33-9.

4. Hyun K-R, Kang S, Lee S. Cost-of-Illness Trends Associated with Thyroid Disease in Korea. Endocrinol Metab. 2014 Sep; 29(3):257-69.

5. Geoff Beckett. UK Thyroid Function Tests Guidelines: Can we manage Thyroid Function Tests in a rational way? https://www.slideshare.net/roger961/uk-thyroidfunction-tests-guidelines

6. .Shalini Gupta,Minni Verma, Ashwani Kumar Gupta,Amandeep Kaur,Vannet Kaur,Kamaljit Singh, Verna M, Ashwani Kumar Gupta, Kaur A, Vannet Kaur, Kamaljit Singh. Are we using Thyroid Function Tests Appropriately? Indian J Clin Biochem. 2011; 26(2):178-81.

7. Kende M, Kandapu S. Evaluation of thyroid stimulating hormone (TSH) alone as a first-line thyroid function test (TFT) in Papua New Guinea. P N G Med J. 2002 Dec;45(3-4):197-9.

8. Gisah Amaral de Carvalho, Perez CLS, Laura Sterian. The clinical use of thyroid function tests. Arq Bras Endocrinol Metab; 2013.

9. Manning P, Cam Kyle, Mike Croxon. Investigating Thyroid Function. bpac better medicine; 2005.

10. Graham H Beastall, Beckett GJ, Jayne Franklyn, William D Fraser, Janis Hickey. UK Guidelines for the Use of Thyroid Function Tests. British Thyroid Association 24-37, 56; 2006.

11. Feldcamp C, Carey JL. An algorithmic approach to thyroid function testing in a managed care setting. 3-year experience. - PubMed - NCBI. Am J Clin Pathol. 1996 Jan; 105(1):11-6.

12. R.Sirkar. Guidelines for the Use of Thyroid Function Tests Grey's Hospital Laboratory. 2006 Aug.

13. CarvalhoI GA de, Camila Luhm Silva Perez (last), Laura Sterian. The clinical use of thyroid function tests. Arq Bras Endocronologia Metabol. 2013; 57.
14. Toubert ME1, Chevret S, Cassinat B, Schlageter $\mathrm{MH}$, Beressi JP, Rain JD. From guidelines to hospital practice: reducing inappropriate ordering of thyroid hormone and antibody tests. I [Internet]. 2000 [cited 2016 Aug 19]. Available from: http://www.ncbi.nlm.nih.gov/pubmed/10822223

15. Mario Skugor. Hypothyroidism and Hyperthyroidism. Cleveland Clinic, Center for Continuing Education; 2014.

16. Kawther El Shafie, Al-Shaqsi A, Badriva AlMarouqi, Lawati BA, Shyam S Ganguly. The Diagnostic Yield of Thyroid Function Tests and their Cost-effectiveness in the Student Clinic at Sultan Qaboos University.mht. Sultan Qaboos UnivMed J. 10(2):215-20.

17. Dennis L Kasper, Anthony S. Fauci, M tephen LH, $\mathrm{d}$, an L. Longo, M. Harrison's principle of internal medicine. 19th ed. Vol. 19. McGraw Hill Education; 2283-2309.

18. Anthony J.Viera. [Thyroid diseases in sub-Saharan Africa]. - PubMed - NCBI [Internet]. 2003 [cited 2016 Aug 4]. Available from: http://www.ncbi.nlm.nih.gov/pubmed/17897900

19. Hyperthyroidism Risk factors - Mayo Clinic [Internet]. [cited 2016 Aug 2]. Available from: http://www.mayoclinic.org/diseasesconditions/hyperthyroidism/basics/riskfactors/con-20020986

20. Vidal-Tirecan G, Toubert ME, Coste G, F P, Durand-Zaleski, and Fulla Y. Reducing the number of T3 orders in the Paris hospital network: towards better appropriateness of thyroid function test prescription. Ann Endocrinol. 2003; 64(3):210-5.

21. Smyth PP, Mark PJ Vander rump Thyroid International: Epidemiology of Thyroid Dysfunction-Hypothyroidism and Hyperthyroidism. Merck KGaADarmstadt; 2009.

22. Thienpont LM, Uytfanghe $\mathrm{K}$ van, Beastall G, Faix JD, Miller TIWG, Nelson JC, et al. Report of the IFCC Working Group for Standardization of Thyroid Function Tests; Part 1: thyroid-stimulating hormone. Clin Chem. 2010 Jun 1; 56(6):902-12.

23. Alexander Werhun, William Hamilton. Are we overusing thyroid function tests? British Journal of General Practice [Internet]. 2013 [cited 2016 Aug 4]. Available from: http://bjgp.org/content/63/613/404.1

24. Ane Garmendia, Madariaga, Palacios SS, Francisco Guillén-Grima, Juan C. Galofré. The Incidence and Prevalence of Thyroid Dysfunction in Europe: A Meta-Analysis. J Clin Endocrinol Metab. 2014 Jan 7; 99:923. 